Pacific Journal of Mathematic 


\title{
SOLVABILITY OF NONLINEAR OPERATOR EQUATIONS
}

\author{
W. J. Cramer, JR. and William O. RaY
}

Let $X$ and $Y$ be Banach spaces, $P: X \rightarrow Y$ a Gateaux differentiable operator having closed graph. Suppose

(i) for each $R>0$ there is a $\delta>0$ such that

$$
d P_{x}(B(0 ; 1)) \supseteqq B(0 ; \delta) \text { whenever }\|x\| \leqq R
$$

(ii) $P^{-1}(K)$ is bounded whenever $\mathrm{cl}(K) \subseteq Y$ is compact; then $P$ is an open mapping of $X$ onto $Y$. Similar results are obtained for compact Gateaux differentiable operators using a local version of (i); the same local version gives a domain invariance theorem for Gateaux differentiable operators having closed graph. Related results deal with M. Altman's theory of contractor directions and theory of normal solvability as developed by F. E. Browder and others.

1. Introduction. Let $X$ and $Y$ be Banach spaces and $P: X \rightarrow Y$ a nonlinear operator. In this paper we consider the global implications of certain local assumptions on $P$ and, in particular, derive general conditions under which $P$ will be an open mapping of $X$ onto $Y$. While our hypotheses are motivated by differentiability conditions on $P$, our results will apply to operators which need not even be continuous.

A 1959 theorem of R.S. Palais [17] provides the prototype for our results; Palais' theorem states that if $P: R^{n} \rightarrow R^{n}$ is a continuously differentiable mapping, then in order for $P$ to be a diffeomorphism it is necessary and sufficient that

0 is not an eigenvalue of $d P_{x}$ for any $x$;

and

$$
\|P x\| \longrightarrow \infty \text { as }\|x\| \longrightarrow \infty .
$$

We are primarily interested in extending the surjectivity portion of Palais' conclusion to operators acting on arbitrary Banach spaces; our methods, in addition, will show the operators we consider are open maps.

Extensions of the above type have recently been obtained for continuously Fréchet differentiable operators $P$ acting on a Banach space $X$ (see $\S 3$ for definitions). In [13] R. Kacurovskii shows that if (1.1) and (1.2) hold and if $(I-P)$ is completely continuous, then $P$ is a homeomorphism of $X$ onto $X$. M. Krasnoselskii has also observed this result is true [16], and in addition has observed that if (1.1) is strengthened to 
$d P_{x}$ is a continuously invertible linear operator for each $x$ and $\sup \left\{\left\|\left(d P_{x}\right)^{-1}\right\|:\|x\| \leqq r\right\}<\infty$ for each $r>0$

then the assumption that $(I-P)$ is completely continuous may be dropped while retaining the conclusion of Kacurovskii's theorem.

In each of the above results, the Inverse Function Theorem implies that $P$ is an open map; a modified Newton-Kantorovich procedure then easily gives surjectivity (see Theorem 4 of [14]) and an elementary covering space argument gives injectivity (see [16]). The arguments rely heavily on continuous differentiability of $P$ and, in this case, the proof of surjectivity is a special case of the contraction mapping principle.

The above results can be thought of as showing that the problem $y=P x$ is well-posed (in the sense of Hadamard) in that they show that

(1.3) the solution $x$ exists for each $y$;

(1.4) the solution is unique;

and

the solution depends continuously on the initial data $y$ (i.e., $P$ is an open mapping).

It will be a consequence of our more basic results that the conclusions (1.3) and (1.5) will hold for Gateaux differentiable operators having closed graph, and also for operators satisfying a coercive condition somewhat weaker than (1.2). In addition, we are able to obtain a domain invariance result for Gateaux differentiable operators which will play much the same role in our theorems as the Inverse Function Theorem plays in the earlier results.

In place of the contraction mapping principle, our basic tool is the following maximal principle of $\mathrm{H}$. Brézis and F. E. Browder [2]:

Proposition B. Let $(X, d, \leqq)$ be a partially ordered metric space and let $\phi: X \rightarrow[0, \infty)$ be an arbitrary function. Suppose

$$
\begin{gathered}
S(x) \equiv\{y \in X: y \geqq x\} \text { is closed for each } x \in X ; \\
x \leqq y \text { and } x \neq y \text { imply } \phi(y)<\phi(x) ; \text { and }
\end{gathered}
$$

any nondecreasing sequence is relatively compact.

Then there is an $x_{0} \in X$ for which $S\left(x_{0}\right)=\left\{x_{0}\right\}$.

The above is striking not only because it includes such well- 
known results as Ekeland's theorem [11] and Caristi's reformulation of the same [10] as easy corollaries, but also because of the elegant simplicity of its proof. For a discussion of related results, the reader is referred to [2] or [12].

We now turn to our results. In $\S 2$ we prove our basic mapping theorem and then derive from this in $\S 3$ our results for Gateaux differentiable operators. We conclude in $\S 4$ with some remarks which relate our results to M. Altman's theory of contractor directions and to the theory of normal solvability.

2. Basic result. In this section we derive our basic mapping result. In $\S 3$ we explore the consequences of this result for the Gateaux differentiable operators, and in $\S 4$ further extend these results to more general mappings.

If $(X, d)$ is a metric space, we denote by $B\left(x ; r^{\circ}\right)$ the set

$$
B(x ; r)=\{w \in X: d(w, x) \leqq r\} .
$$

Also, if $P: D \subseteq X \rightarrow Y$, where $Y$ is a Banach space, we say $P$ has closed graph if whenever $x_{n} \rightarrow x_{0}$ in $X$ and $P x_{n} \rightarrow y_{0}$ in $Y$, then $x_{0} \in D$ and $P\left(x_{0}\right)=y_{0}$.

Theorem 2.1. Let $(X, d)$ be a complete metric space, $D \subseteq X, Y$ a Banach space, $P: D \rightarrow Y$ a mapping having closed graph and $B:[0, \infty) \rightarrow[0, \infty)$ a continuous nondecreasing function satisfying $\int_{0}^{b} s^{-1} B(s) d s<\infty$ for each $b>0$. Suppose for some fixed $w \in D$ and constants $q \in(0,1), \alpha>0, M>0$ and $a \equiv M\|P w\| e^{1-q}$ that

$$
(1-q)^{-1} \int_{0}^{a} s^{-1} B(s) d s \leqq \alpha .
$$

Suppose further that, corresponding to each $x \in B(w ; \alpha) \cap D$ there is an $\bar{x} \in D$ and an $\varepsilon \in(0,1]$ such that

$$
\|P \bar{x}-(1-\varepsilon) P x\| \leqq q \varepsilon\|P x\| ;
$$

and

$$
d(\bar{x}, x) \leqq \varepsilon B(M\|P x\|) .
$$

Then there is an $x_{0} \in B(w ; \alpha) \cap D$ for which $P x_{0}=0$.

Proof. Set $E=D \times[0, \infty)$, define a metric $\rho$ on $E$ by

$$
\rho((x ; s),(y, t))=\max \{|t-s|, d(x, y),\|P x-P y\|\},
$$

and define $\phi: E \rightarrow[0, \infty)$ by $\phi(x, s)=\|P x\|$. Since $P$ has closed graph, $E$ is a complete metric space and $\phi:(E, \rho) \rightarrow[0, \infty)$ is continuous. 
We now define a partial ordering on $E$ by saying $(x, s) \leqq(y, t)$ if and only if

$$
\begin{gathered}
s \leqq t ; \\
\|P x-P y\| \leqq \frac{1+q}{1-q}(\|P x\|-\|P y\|) \\
\|P y\| \leqq\|P x\| e^{-(1-q)(t-s)} ;
\end{gathered}
$$

and

$$
d(x, y) \leqq \int_{s}^{t} B\left(M\|P x\| e^{1-q} e^{(1-q)(s-u)}\right) d u .
$$

It is clear that " $\leqq$ " is antisymmetric and reflexive, and that (2.4), (2.5) and (2.6) are transitive. To see that (2.7) is transitive as well, suppose $(x, s) \leqq(y, t) \leqq(z, r)$ and apply (2.7) and (2.6):

$$
\begin{aligned}
d(x, z) & \leqq d(x, y)+d(y, z) \\
& \leqq \int_{s}^{t} B\left(M\|P x\| e^{1-q} e^{(1-q)(s-u)}\right) d u+\int_{t}^{r} B\left(M\|P y\| e^{1-q} e^{(1-q)(t-u)}\right) d u \\
& \leqq \int_{s}^{t} B\left(M\|P x\| e^{1-q} e^{(1-q)(s-u)}\right) d u+\int_{t}^{r} B\left(M\|P x\| e^{1-q} e^{(1-q)(s-u)}\right) d u \\
& =\int_{s}^{r} B\left(M\|P x\| e^{(1-q)(s-u)}\right) d u,
\end{aligned}
$$

so $(x, s) \leqq(z, r)$.

Next, for $(x, s) \in E$, define

$$
S(x, s)=\{(y, t) \in E:(y, t) \geqq(x, s)\}
$$

and observe that $S(x, s)$ is a closed subset of $(E, \rho)$. Also observe that (2.6), (2.7) and (2.1) imply that $S(w ; 0) \subseteq B(w ; \alpha)$ : if $(x, s) \in S(w, 0)$ then

$$
\begin{aligned}
d(x, w) & \leqq \int_{0}^{s} B\left(M\|P(w)\| e^{1-q} e^{-(1-q) u}\right) d u \\
& \leqq(1-q)^{-1} \int_{0}^{a} v^{-1} B(v) d v \leqq \alpha
\end{aligned}
$$

making the change of variables $v=M\|P w\| e^{1-q} e^{-(1-q) u}$.

Now set $S \equiv S(w ; 0)$; it will suffice to show that $P$ has a zero in $S$. Thus we suppose $P x \neq 0$ for all $x \in B(w ; \alpha)$ and apply the Brézis-Browder maximal principle to obtain a contradiction. Notice $\phi: S \rightarrow[0, \infty)$ and, by 2.7 and 2.6 , if $(x, s) \leqq(y, t)$ and $(x, s) \neq(y, t)$, then $\phi(y, t)<\phi(x, s)$. Also, if $(x, s) \in S$, then $S(x, s)$ is a closed set and is a subset of $S$ by transitivity of “ $\leqq$ ".

All that remains before applying Proposition B is to verify that, 
if $\left\{\left(x_{n}, t_{n}\right)\right\} \subseteq S$ is a nondecreasing sequence, then $\left\{\left(x_{n}, t_{n}\right)\right\}$ is precompact. Since each $\left(x_{n}, t_{n}\right) \in S$, (2.6) implies

$$
\left\|P\left(x_{n}\right)\right\| \leqq\|P(w)\| e^{-(1-q) t_{n}} .
$$

Now fix $n<m$; we will use (2.8) to show that $\left\{x_{n}\right\}$ is a Cauchy sequence in $(X, d)$ :

$$
\begin{aligned}
& d\left(x_{n}, x_{m}\right) \leqq \sum_{k=n}^{m-1} d\left(x_{k+1}, x_{k}\right) \\
& \leqq \sum_{k=n}^{m-1} \int_{t_{k}}^{t_{k+1}} B\left(M\left\|P x_{k}\right\| e^{1-q} e^{(1-q)\left(t_{k}-u\right)}\right) d u \\
& \leqq \sum_{k=n}^{m-1} \int_{t_{k}}^{t_{k+1}} B\left(M\|P w\| e^{1-q} e^{-(1-q) u}\right) d u \\
&=\int_{t_{n}}^{t_{m}} B\left(M\|P w\| e^{1-q} e^{-(1-q) u}\right) d u, \text { i.e., } \\
& d\left(x_{n}, x_{m}\right) \leqq \int_{t_{n}}^{t_{m}} B\left(M\|P w\| e^{1-q} e^{-(1-q) u}\right) d u
\end{aligned}
$$

Now recall $\left\{t_{n}\right\}$ is a nondecreasing sequence, and consider the two cases:

I. $t_{n} \rightarrow \infty$ as $n \rightarrow \infty$. Making the change of variables $v=$ $M\|P w\| e^{1-q} e^{-(1-q) u}$ in 2.9 gives

$$
d\left(x_{n}, x_{m}\right) \leqq(1-q)^{-1} \int_{a_{m}}^{a_{n}} v^{-1} B(v) d v
$$

where $a_{k}=M\|P w\| e^{1-q} e^{-(1-q) t_{k}}$. Since $a_{n}, a_{m} \rightarrow 0$ as $n, m \rightarrow \infty$ and since $\int_{0}^{b} v^{-1} B(v) d v<\infty$ for all $b>0$, it follows that $\left\{x_{n}\right\}$ is a Cauchy sequence in $(X, d)$.

II. $t_{n} \rightarrow t_{\infty}$ as $n \rightarrow \infty$. Once again, the right side of (2.10) goes to zero as $n, m \rightarrow \infty$ and thus $\left\{x_{n}\right\}$ is Cauchy.

We next show that $\left\{P x_{n}\right\}$ is a Cauchy sequence in $Y$. By (2.5), $\left\{\left\|P x_{n}\right\|\right\}$ is a nonincreasing sequence and thus convergent, so, for $n<m$,

$$
\begin{aligned}
\left\|P x_{n}-P x_{m}\right\| & \leqq \sum_{k=n}^{m-1}\left\|P\left(x_{k+1}\right)-P\left(x_{k}\right)\right\| \\
& \leqq \frac{1+q}{1-q}\left(\left\|P x_{n}\right\|-\left\|P x_{m}\right\|\right)
\end{aligned}
$$

and thus $\left\{P x_{n}\right\}$ is a Cauchy sequence in $Y$.

Since $\left\{x_{n}\right\} \subseteq B(w ; \alpha)$ and both $\left\{x_{n}\right\}$ and $\left\{P x_{n}\right\}$ are Cauchy sequences, it follows from the assumption that $P$ has closed graph that there is an $x_{\infty} \in B(w ; \alpha)$ such that $x_{n} \rightarrow x_{\infty}$ and $P x_{n} \rightarrow P x_{\infty}$ as $n \rightarrow \infty$. Now if $t_{n} \rightarrow \infty$ as $n \rightarrow \infty$, then (2.6) implies 


$$
\left\|P x_{\infty}\right\|=\lim _{n \rightarrow \infty}\left\|P x_{n}\right\| \leqq \lim _{n \rightarrow \infty}\left\|P_{w}\right\| e^{-(1-q) t_{n}}=0,
$$

i.e., $P x_{\infty}=0$, contrary to our assumption. Thus there is a $t_{\infty} \in[0, \infty)$ such that $t_{n} \rightarrow t_{\infty}$ as $n \rightarrow \infty$. Since $S$ is closed, $\left(x_{\infty}, t_{\infty}\right) \in S$ and we have shown nondecreasing sequences in $S$ are convergent, more than was required.

Now by Proposition B there is an $\left(x_{0}, t_{0}\right) \in S$ for which $S\left(x_{0}, t_{0}\right)=$ $\left\{\left(x_{0}, t_{0}\right)\right\}$. Choose $\bar{x}_{0} \in D$ and $\varepsilon \in(0,1]$ in accordance with (2.2) and (2.3). We will show that $\left(\bar{x}_{0}, t_{0}+\varepsilon\right) \geqq\left(x_{0}, t_{0}\right)$, from which $\left(\bar{x}_{0}, t_{0}+\varepsilon\right)=$ $\left(x_{0}, t_{0}\right)$, an obvious contradiction.

Rewriting (2.2), we obtain

$$
\left\|P \bar{x}_{0}-P x_{0}\right\| \leqq(1+q) \varepsilon\left\|P x_{0}\right\|
$$

and

$$
\left\|P \bar{x}_{0}-P x_{0}\right\| \leqq(1-(1-q) \varepsilon)\left\|P x_{0}\right\| .
$$

Combining the above yields

$$
\left\|P \bar{x}_{0}-P x_{0}\right\| \leqq \frac{1+q}{1-q}\left(\left\|P x_{0}\right\|-\left\|P \bar{x}_{0}\right\|\right),
$$

so (2.5) holds. Moreover, (2.11) and the MacLaurin's Series for $e^{t}$ imply

$$
\left\|P \bar{x}_{0}\right\| \leqq\left\|P x_{0}\right\| e^{-\{1-q) \varepsilon},
$$

so (2.6) holds. Finally, we derive (2.7) from (2.3) and the fact that $\varepsilon \in(0,1]:$

$$
\begin{aligned}
d\left(\bar{x}_{0}, x_{0}\right) & \leqq \varepsilon B\left(M\left\|P x_{0}\right\|\right) \\
& =\int_{0}^{\varepsilon} B\left(M\left\|P x_{0}\right\| e^{0}\right) d u \\
& \leqq \int_{0}^{\varepsilon} B\left(M\left\|P x_{0}\right\| e^{(1-q)(1-u)}\right) d \imath \\
& =\int_{t_{0}}^{t_{0}+\varepsilon} B\left(M\left\|P x_{0}\right\| e^{1-q} e^{(1-q)\left(t_{0}-u\right)}\right) d u
\end{aligned}
$$

and thus (2.7) holds.

3. Gateaux differentiable operators. Let $X$ and $Y$ be Banach spaces and $P: X \rightarrow Y$ a (nonlinear) operator. We call $P$ Gateaux differentiable at $x$ if there is a function (called the Gateaux Variation of $P$ ) $d P_{x}: X \rightarrow Y$ such that, for each $y \in X$

$$
\lim _{t \rightarrow 0} \frac{P(x+t y)-P(x)}{t}=d P_{x}(y) .
$$


If $d P_{x}$ is a bounded linear operator and if the limit (3.1) is attained uniformly for $y \in B(0 ; 1)$, then $P$ is Fréchet-differentiable. If the $\operatorname{map} x \rightarrow d P_{x}$ is continuous from $X$ to the bounded linear operators mapping $X$ to $Y$, then $P$ is continuously Fréchet differentiable. Easy examples of mappings from $R^{2}$ to $R^{1}$ show that Gateaux differentiable operators need not be continuous, that the Gateaux variation need not be linear and, even when it is linear, need not be a Fréchet derivative. It is clear, however, that the Gateaux variation is homogeneous, i.e., $d P_{x}(\lambda y)=\lambda d P_{x}(y)$ for all $\lambda \in R$.

The following lemma provides the basic link between this section and $\S \S 2$ and 4 of this paper.

Lemma 3.1. Let $P: X \rightarrow Y$ be Gateaux differentiable at $x$ and suppose, for some $\delta>0$,

$$
d P_{x}(B(0 ; 1)) \supseteqq B\left(0 ; \delta^{-1}\right) .
$$

Then for each $y \in Y$ there is an $\bar{x} \in X$ and $\varepsilon \in(0,1]$ for which

$$
\|P \bar{x}-P x-\varepsilon(y-P x)\| \leqq \frac{\varepsilon}{2}\|y-P x\| ;
$$

and

$$
\|\bar{x}-x\| \leqq \delta \varepsilon\|y-P x\|
$$

We remark that if $P$ is Fréchet differentiable and $d P_{x}$ is invertible, then we may take $\delta=\left\|\left(d P_{x}\right)^{-1}\right\|$, and the hypotheses of the lemma are fulfilled. The assumption (3.2) will replace invertibility of $d P_{x}$ in the results of Kacurovskii and Krasnoselskii.

Proof of Lemma 3.1. First observe that if $y=P x$, we may take $\bar{x}=x$ and let $\varepsilon$ be arbitrary; thus without loss of generality we suppose that $y-P x \neq 0$. Set $w=\delta^{-1}\|y-P x\|^{-1}(y-P x)$, so $w \in B\left(0 ; \delta^{-1}\right)$. By $(3.2)$, there is a $v \in B(0 ; 1)$ such that $d P_{x}(v)=w$, so if $\bar{v}=\delta\|y-P x\| v, d P_{x}(\bar{v})=(y-P x)$. By Gateaux differentiability of $P$, there is an $\varepsilon \in(0,1]$ such that

$$
\frac{1}{\varepsilon}\left\|P(x+\varepsilon \bar{v})-P(x)-\varepsilon d P_{x}(\bar{v})\right\| \leqq \frac{1}{2}\|y-P x\| .
$$

Taking $\bar{x}=x+\varepsilon \bar{v}$, we obtain from the above that

$$
\|P(\bar{x})-P(x)-\varepsilon(y-P(x))\| \leqq \frac{\varepsilon}{2}\|y-P x\|
$$

so (3.3) holds. Also, since $\|v\| \leqq 1$, 


$$
\|\bar{x}-x\|=\varepsilon\|\bar{v}\| \leqq \varepsilon \delta\|y-P x\|
$$

so (3.4) holds as well.

We begin by proving a domain invariance result which can be thought of as an "inverse function theorem" for Gateaux differentiable operators.

Theorem 3.2. Let $X$ and $Y$ be Banach spaces, $P: X \rightarrow Y$ a Gateaux differentiable operator having closed graph and $U \subseteq X$ an open set. Suppose for each $w \in U$ there is a $\delta(w)>0$ and $\alpha(w)>0$ such that, if $\|x-w\| \leqq \alpha(w)$ then $d P_{x}(B(0 ; 1)) \supseteqq B\left(0 ;(\delta(w))^{-1}\right)$. Then $P(U)$ is an open set in $Y$.

Proof. Fix $w \in U$ and choose $\alpha \leqq \alpha(w)$ so small that $B(w ; \alpha) \subseteq$ $U$; set $M=\delta(w)$ and $\hat{\delta} \equiv \alpha\left(2 M e^{1 / 2}\right)^{-1}$. It will suffice to show $P(B(w ; \alpha)) \supseteqq B(P(w) ; \hat{\delta})$. To this end, fix $y \in B(P(w) ; \hat{\delta})$ and set $\bar{P}(x)=y-P(x)$; it suffices to show there is an $x_{0} \in B(w ; \alpha)$ such that $\bar{P}\left(x_{0}\right)=0$. Observe that

$$
2\|\bar{P}(w)\| M e^{1 / 2} \leqq 2 \widehat{\delta} M e^{1 / 2} \leqq \alpha
$$

and so (2.1) of Theorem 2.1 holds with $B(s)=s, q=1 / 2$ and $M=\delta(w)$. Moreover, by Lemma 3.1 , for each $x \in B(w ; \delta)$ there is an $\bar{x} \in X$ and an $\varepsilon \in(0,1]$ such that

$$
\|\bar{P} \bar{x}-(1-\varepsilon) \bar{P}(x)\| \leqq \frac{\varepsilon}{2}\|\bar{P}(x)\|
$$

and

$$
\|\bar{x}-x\| \leqq M \varepsilon\|\bar{P}(x)\|
$$

so that (2.2) and (2.3) hold. Theorem 2.1 then gives a zero for $\bar{P}$ in $B(w ; \alpha)$, and thus $P(B(w ; \alpha)) \supseteqq B(P(w) ; \widehat{\delta})$.

The above theorem easily yields our extensions of the results of Kacurovskii and Krasnoselskii.

Theorem 3.3. Let $X$ be a Banach space, $P: X \rightarrow X$ a Gateaux differentiable operator having closed graph and suppose $(I-P)$ is a compact operator (i.e., $I-P$ sends bounded sets to precompact sets). Suppose for each $w \in X$ there is an $\alpha(w)>0$ and $a \delta(w)>0$ such that if $\|x-w\| \leqq \alpha(w)$ then $d P_{x}(B(0 ; 1)) \supseteqq B\left(0 ;(\delta(w))^{-1}\right)$. Suppose also

(3.5) $P^{-1}(K)$ is bounded whenever $\mathrm{cl}(K)$ is compact. Then $P$ is an open mapping of $X$ onto $X$. 
In Kacurovskii's result, it is assumed that $P$ is continuously Fréchet differentiable, $(I-P)$ is completely continuous, and that 0 is not an eigenvalue of $d P_{x}$ for any $x$. In this case, $\left(I-d P_{x}\right)$ is a compact linear operator (see, for example, Schwartz [20], Theorem 1.40) and 1 is not an eigenvalue of $\left(I-d P_{x}\right)$. The Fredholm alternative then implies $d P_{x}$ is continuously invertible for each $x$. Since the collection of invertible operators in $B L(X)$ (the bounded linear operators acting on $X)$ is open in $B L(X)$ and $P$ is continuously differentiable, it follows that, for fixed $w$, we may choose $\alpha(w)$ so small that

$$
\sup \left\{\left\|\left(d P_{x}\right)^{-1}\right\|:\|x-w\| \leqq \alpha(w)\right\} \equiv \delta(w)<\infty,
$$

and hence the assumption that $d P_{x}(B(0 ; 1)) \supseteqq B\left(0 ;(\delta(w))^{-1}\right)$ is fulfilled. Clearly the assumption (3.5) is more general, in infinite dimensions, than (1.2).

Proof of Theorem 3.3. By Theorem 3.2, $P(X)$ is open; thus it suffices to show $P(X)$ is closed. Let $\left\{x_{n}\right\} \subseteq X$ satisfy $P x_{n} \rightarrow y$ for some $y \in Y$. Since $\left\{P x_{n}\right\}$ is precompact, $\left\{x_{n}\right\}$ is bounded, and so $\left\{x_{n}-P x_{n}\right\}$ is precompact. By passing to subsequences, we may suppose that $x_{n}-P x_{n} \rightarrow x_{0}$ as $n \rightarrow \infty$. Since $P x_{n} \rightarrow y$, it follows that $x_{n} \rightarrow x_{0}+y$ and, since $P$ has closed graph, that $P\left(x_{0}+y\right)=y$, establishing that $P$ has closed range.

We remark that if one weakens the assumption that $\|x-w\| \leqq$ $\alpha(w)$ implies $d P_{x}(B(0 ; 1)) \supseteqq B\left(0 ;(\delta(w))^{-1}\right)$ to the assumption that each $d P_{x}$ have dense range, then the conclusion that $P$ is surjective remains valid. The proof is an easy application of Caristi's theorem (see, for example, [15]).

We next turn to Krasnoselskii's result.

TheORem 3.4. Let $X$ and $Y$ be Banach spaces, $P: X \rightarrow Y a$ Gateaux differentiable operator having closed graph, and suppose (3.5) holds. Suppose in addition that

(3.6) For each $R \geqq 0$ there is a $\delta(R)>0$ such that if $\|x\| \leqq R$ then $d P_{x}(B(0 ; 1)) \supseteqq B\left(0 ;(\delta(R))^{-1}\right)$.

Then $P$ is an open mapping of $X$ onto $Y$.

Condition (3.6) is again a (nonlinear) extension of the condition $\left(1.1^{\prime}\right)$.

Proof of Theorem 3.4. As before, Theorem 3.2 implies that $P$ is an open mapping and hence it suffices to show that $P$ has closed range. Thus we choose $y \in Y$ and $\left\{x_{n}\right\} \subseteq X$ such that $P x_{n} \rightarrow y$. 
From condition (3.5), $\left\{x_{n}\right\}$ is a bounded sequence and thus we may choose $R>0$ so that $B\left(x_{n} ; 1\right) \leqq B(0 ; R)$ for each $n$. Moreover, we may choose $n$ so large that

$$
2\left\|P x_{n}-y\right\| \delta(R) e^{1 / 2} \leqq 1 .
$$

Setting $\bar{P}(x)=y-P x$, we again have (2.1) fulfilled, this time with $\alpha=1, M=\delta(R)$ and $q=1 / 2$. Lemma 3.1 again implies that (2.2) and (2.3) hold for $\bar{P}$, and thus gives a zero for $\bar{P}$. This shows that $y \in P(X)$, and thus that $P(X)=Y$.

We list here one final corollary for Gateaux differentiable operators.

Theorem 3.5. Let $X$ and $Y$ be Banach spaces, $P: X \rightarrow Y$ a Gateaux differentiable operator having closed graph and suppose there is a $\delta>0$ such that for each $x \in X d P_{x}(B(0 ; 1)) \supseteqq B(0 ; \delta)$. Then $P$ is an open mapping of $X$ onto $Y$.

The surjectivity conclusion of this theorem was proved in [19] for Fréchet differentiable operators; the proof extends with little change to this slightly more general setting. The domain invariance is, of course, an immediate consequence of Theorem 3.2.

Finally, we remark that the sequences considered in the proof of Theorem 2.1 are, in the context of Fréchet differentiable operators, iterations of the $\operatorname{map} x: \rightarrow x-\varepsilon\left(d P_{x}\right)^{-1}(P x)$ where $\varepsilon \in(0,1]$. In the case $\varepsilon=1$, the resulting sequences reduce to the Newton-Kantorovich iteration for locating zeros of $P$. In this sense, the Brézis-Browder principle provides an abstract Newton-Kantorovich scheme for solving nonlinear equations (although, in general, the proofs are not constructive).

4. Contractor directions and normal solvability. In a series of fascinating papers M. Altman has developed a generalized notion of differentiability which is essentially based on the same idea as in our Lemma 3.1 in the case that $d P_{x}$ is an invertible linear operator. Altman's theory, which is extensively described in his book [1], is predicated on his concept of contractor directions; our remarks below are based on this reference. (We give here a some. what more general formulation of contractor directions than that which appears in Chapter 5 of [1].)

Let $X$ be a complete metric space, $D \leqq X, Y$ a Banach space and $P: D \rightarrow Y$. In addition let $g: D \rightarrow[0, \infty)$ be an arbitrary function, $q \in(0,1)$ and $B:[0, \infty) \rightarrow[0, \infty)$ a continuous nondecreasing function for which 


$$
\int_{0}^{b} s^{-1} B(s) d s<\infty
$$

for all $b>0$. We will call $P$ A-differentiable if, for each $y \in Y$ and for each $x \in D$ there is an $\bar{x} \in D$ and an $\varepsilon \in(0,1]$ for which

$$
\|P \bar{x}-P x-\varepsilon y\| \leqq q \varepsilon\|y\|
$$

and

$$
d(\bar{x}, x) \leqq \varepsilon B(g(x)\|y\|)
$$

The above definition differs slightly from Altman's " $B-g$ differentiability" (see pg. 116 of [1]) in two ways. First he assumes $g:[0, \infty) \rightarrow[0, \infty)$ is continuous and replaces $(4.2)$ with

$$
d(\bar{x}, x) \leqq \varepsilon B(g(\|x\|)\|y\|) \text {. }
$$

Secondly, instead of assuming (4.1) and (4.2') hold for all $y \in Y$ and $x \in X$, he denotes by $\Gamma(x)$ the collection of all $y \in Y$ for which there exists an $\bar{x} \in D$ and $\varepsilon \in(0,1]$ such that $(4.1)$ and $\left(4.2^{\prime}\right)$ hold $(\Gamma(x)$ is then called the contractor direction set for $P$ at $x$ ). Basic to most of his results, however, is the condition that $\Gamma(x)=Y$ for all $x \in X$ (see, for example, Lemma 1.1, pg. 88 or Lemma 2.1, pg. 92 of [1]) and thus the $A$-differentiable maps include the essential features of the $B-g$ differentiable maps. Lemma 3.1 clearly supplies the motivation for the above definitions: If $X$ is a Banach space, $P$ is Fréchet differentiable and $d P_{x}$ is continuously invertible for all $x$, then (4.1) and (4.2) hold with $B(s)=s$ and $g(x)=\left\|\left(d P_{x}\right)^{-1}\right\|$. The results of $\S 3$ can easily be reformulated in this context. There is no significant variation in the proofs, and so we omit them.

TheOREM 4.1. Let $X, D, Y, q, B, g$ and $P$ be as above, and suppose $P: D \rightarrow Y$ is A-differentiable and has closed graph. Suppose for each $w \in D$ there is a $\delta>0$ and an $M>0$ such that $d(w, x) \leqq \delta$ and $x \in D$ implies $g(x) \leqq M$. Then $P(D)$ is an open subset of $Y$.

Theorem 4.2. Let $X, D, Y, q, B, g$ and $P$ be as above, and suppose $P: D \rightarrow Y$ is $A$-differentiable and has closed graph. Suppose $X$ is a Banach space and for each $R>0$

$$
\sup \{g(x):\|x\| \leqq R, x \in D\}<\infty
$$

and that $P^{-1}(K)$ is bounded whenever $\mathrm{cl}(K)$ is compact. Then $P$ is an open mapping of $D$ onto $Y$.

The surjectivity portion of Theorem 4.2 appears in [1] (Theorem 1.3, pg. 125) for $B-g$ differentiable maps; notice we make sub- 
stantially weaker assumptions on the mapping $g$. We also observe that Altman's proof of his result (which predates the Brézis-Browder principle) relies on an intricate transfinite induction scheme which our technique avoids altogether. In addition, our technique yields the new domain invariance principle embodied in Theorem 4.1.

We conclude with some remarks relating the above results with the theory of normal solvability. Let $X$ and $Y$ be Banach spaces $A \in B L(X, Y)$, and let $A^{*}$ denote the adjoint mapping from $Y^{*}$ to $X^{*}$. If $A$ has closed range, then a classical result of Hausdorff [13] states that $A(X)=\left(N\left(A^{*}\right)\right)^{\perp}$, the annihilator in $Y$ of the null space of $A^{*}$; in this case the linear equation $A x=y$ is called normally solvable.

The nonlinear case was first considered by Pohozhayev [18], who assumed that $P: X \rightarrow Y$ is a continuously Gateaux differentiable operator with weakly closed range satisfying

$$
\left\|P x-y_{0}\right\|=\operatorname{dist}\left(y_{0} ; P(X)\right) \Longrightarrow y_{0}-P(x) \in\left(N\left(d f_{x}^{*}\right)\right)^{\perp},
$$

and showed that this condition is sufficient to guarantee $y_{0} \in P(X)$. If $P$ is linear and $Y$ is reflexive, this reduces to Hausdorff's result.

In a series of papers [3-9], Browder has considerably sharpened and generalized Pohozhayev's result. He supposes $X$ is a topological space, $P: X \rightarrow Y$ and defines [4] the asymptotic direction set of $P$ at $x$ by

$$
D(x)=\bigcap_{\varepsilon>0} \operatorname{cl}(D(x ; \varepsilon))
$$

where

$$
D(x ; \varepsilon)=\{y \in Y: y=\xi(P(u)-P(x)), u \in X, \xi \geqq 0,\|P(u)-P(x)\|<\varepsilon\} .
$$

He then shows $[6,8]$ that sufficient conditions for $y \in P(X)$ are $P$ has closed range and: there exist $r>0$ and $q<1$ such that

$$
B(y ; r) \cap P(X) \neq \varnothing ;
$$

and

$$
\text { if } P(x) \in B(y ; r) \text { then } \operatorname{dist}(y-P(x), D(x)) \leqq q\|y-P(x)\| .
$$

He also shows that if $P$ is continuously Gateaux differentiable, then $\left(N\left(d P_{x}^{*}\right)\right)^{\perp} \subseteq D(x)$ (Proposition 1 of [6]) and hence (4.4) follows from (4.5) and (4.6) upon taking $r=\operatorname{dist}(y ; P(X))$.

In their paper [15], Kirk and Caristi show that (4.6) can be further weakened to

for each $w \in B(y ; r) \cap P(X)$ with $w \neq y$ there is a $u \in P(X)$ and $\xi \geqq 1$ such that $\|\xi(u-w)-(y-w)\| \leqq q\|w-y\|$ 
(that (4.6) implies (4.7) is a routine consequence of the definition).

We now observe that (4.7) is the same as (4.1), except for the additional condition $w \in B(y ; r)$. Since it follows readily from (4.7) that $\|u-y\| \leqq r$ (see (2.3) of [15]), we observe that (4.1) and (4.7) are essentially the same, and thus both Theorems 4.1 and 4.2 can be regarded as normal solvability results of the same type as those of Browder. In this sense, these results are a continuation of Browder's development of the theory of normal solvability.

The authors are indebted to Professor L. Talman for bringing [16] to their attention and for somewhat sharpening their statement of Theorem 3.3.

Added in proof. I. Ekeland has recently informed us [21] that some of the results of $\S 4$ of this paper (in particular, the surjectively conclusion of Theorem 4.2) have been independently obtained by G. Lebourg. In addition, Lebourg has constructed examples relating to the necessity of some of the hypotheses of this theorem.

\section{REFERENCES}

1. M. Altman, Contractors and contractor directions theory and applications, Marcel Dekker, Inc., New York, 1977.

2. H. Brezis and F. E. Browder, A general principle on ordered sets in nonlinear functional analysis, Advances in Math., 21 (1976), 355-364.

3. F. E. Browder, On the Fredholm alternative for nonlinear operators, Bull. Amer. Math. Soc., 76 (1970), 993-998.

4. - Normal solvability for nonlinear mappings in Banach spaces, Bull. Amer. Math. Soc., 77 (1971), 73-77.

5. - Normal solvability and the Fredholm alternative for mappings in infinite dimensional monifolds, J. Functional Analysis, 8 (1971), 250-274.

6. Normal solvability for nonlinear mappings and the geometry of Banach spaces, "Problems in Nonlinear Analysis," pp. 37-66, Edizioni Cremonese, Roma, Italy, 1971.

7. - Normal solvability and existence theorems for nonlinear mappings in Banach spaces, "Problems in Nonlinear Analysis," pp. 19-35, Edizioni Cremonese, Roma, Italy, 1971.

8. - Normal solvability and $\phi$-accretive mappings of Banach spaces, Bull. Amer. Math. Soc., 78 (1972), 196-192.

9. Normally solvable nonlinear mappings in Banach spaces and their homotopies, J. Functional Analysis, 17 (1974), 441-446.

10. J. Caristi, Fixed point theorems for mappings satisfying inwardness conditions, Trans. Amer. Math. Soc., 215 (1976), 241-251.

11. I. Ekeland, Sur les problemes variationnels, Compte Rendus Acad. Sci. Paris, 275 (1972), 1057-1059.

12. Nonconvex minimization problems, Bull. Amer. Math. Soc., 1 (1979), 443-474.

13. F. Hausdorff, Zur theorie der linearen metrischen Raume, Jour. Reine. Angew. Math., 167 (1932), 294-311.

14. R. I. Kacurowskii, Generalization of the Fredholm theorems and of theorems on linear operators with closed range to some classes of nonlinear operators, Dokl. Acad. 
Nauk SSSR, 197 (1971), 520-523=Soviet Math. Dokl., 12 (1971), 168-172.

15. W. A. Kirk and J. Caristi, Mapping theorems in metric and Banach spaces, Bull. Acad. Polon. Sci. ser. Sci. Math. Astronom. Phys., 23 (1975), 891-894.

16. M. A. Krasnosel'skii, On several new fixed point principles, Dokl. Akad. Nauk SSSR, 14 (1973), 259-261.

17. R. S. Palais, Natural operations on differential forms, Trans. Amer. Math. Soc., 92 (1959), 125-141.

18. S. I. Pohozhayev, On the normal solvability of nonlinear operators, Dokl. Akad. Nauk SSSR, 184 (1969), 40-43.

19. I. Rosenholtz and W. O. Ray, Mapping theorems for differentiable operators, preprint.

20. J. T. Schwartz, Nonlinear functional analysis, Gordon and Breach, New York, 1969.

21. I. Ekeland, Private communication, dated 22 October 1980.

Received April 7, 1980. Research of the second author was supported in part by a grant from the ISU Science and Humanities Research Institute.

Iowa State University

Ames, IA 50011

Current address of second author: The University of Oklahoma, Norman, OK 73019. 


\section{PACIFIC JOURNAL OF MATHEMATICS}

\section{EDITORS}

DONALD BABBITT (Managing Editor)

University of Galifornia

Los Angeles, California 90024

Hugo RossI

University of Utah

Salt Lake City, UT 84112

C. C. MOORE AND ANDREW OGG

University of California

Berkeley, CA 94720
J. DugundJI

Department of Mathematics University of Southern California Los Angeles, California 90007

R. FinN and J. Milgram Stanford University Stanford, California 94305

\section{ASSOCIATE EDITORS}

R. ARENS

E. F. BeCKenbaCh

B. H. NEUManN

F. WOLF

K. YosHIDA

\section{SUPPORTING INSTITUTIONS}

UNIVERSITY OF ARIZONA

UNIVERSITY OF BRITISH COLUMBIA

CALIFORNIA INSTITUTE OF TECHNOLOGY

UNIVERSITY OF CALIFORNIA

MONTANA STATE UNIVERSITY

UNIVERSITY OF NEVADA, RENO

NEW MEXICO STATE UNIVERSITY

OREGON STATE UNIVERSITY
UNIVERSITY OF OREGON

UNIVERSITY OF SOUTHERN CALIFONIA

STANFORD UNIVERSITY

UNIVERSITY OF HAWAII

UNIVERSITY OF TOKYO

UNIVERSITY OF UTAH

WASHINGTON STATE UNIVERSITY

UNIVERSITY OF WASHINGTON 


\section{Pacific Journal of Mathematics}

\section{Vol. 95, No. $1 \quad$ September, 1981}

John Allen Beachy and William David Blair, On rings with bounded

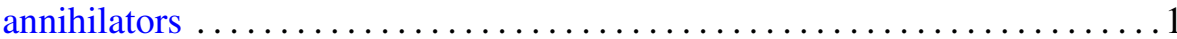

Douglas S. Bridges, A constructive look at positive linear functionals on

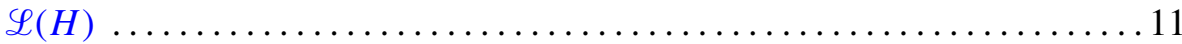

Muneo Chō and Makoto Takaguchi, Boundary points of joint numerical ranges

W. J. Cramer and William O. Ray, Solvability of nonlinear operator equations

Lester Eli Dubins and Gideon Schwarz, Equidiscontinuity of

Borsuk-Ulam functions

Maria Fragoulopoulou, Spaces of representations and enveloping 1.m.c.

*-algebras

Robert F. Geitz and J. Jerry Uhl, Jr., Vector-valued functions as families of scalar-valued functions

Ross Geoghegan, The homomorphism on fundamental group induced by a homotopy idempotent having essential fixed points

Ross Geoghegan, Splitting homotopy idempotents which have essential fixed points

Paul Jacob Koosis, Entire functions of exponential type as multipliers for

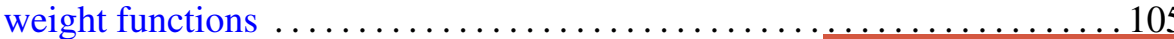

David London, Monotonicity of permanents of certain doubly stochastic matrices

Howard J. Marcum, Two results on cofibers

Giancarlo Mauceri, Zonal multipliers on the Heisenberg group

Edward Wilfred Odell, Jr. and Y. Sternfeld, A fixed point theorem in $c_{0} \quad \ldots 161$

Bernt Karsten Oksendal, Brownian motion and sets of harmonic measure zero

Andrew Douglas Pollington, The Hausdorff dimension of a set of normal numbers

Joe Repka, Base change lifting and Galois invariance ...

Gerald Suchan, Concerning the minimum of permanents on doubly stochastic circulants

Jun-ichi Tanaka, On isometries of Hardy spaces on compact abelian groups

Aaron R. Todd, Quasiregular, pseudocomplete, and Baire spaces 